\title{
Truncating mutation in TANC2 in a Chinese boy associated with Lennox-Gastaut syndrome: a case report
}

\author{
Yang Tian ${ }^{\dagger}$, Zhen Shi ${ }^{\dagger}$, Chi Hou ${ }^{\dagger}$, Wenjuan Li, Xiuying Wang, Haixia Zhu, Xiaojing Li and Wen-Xiong Chen * (0)
}

\begin{abstract}
Background: Lennox-Gastaut syndrome (LGS) is a severe epileptic encephalopathy that can be caused by brain malformations or genetic mutations. Recently, genome-wide association studies have led to the identification of novel mutations associated with LGS. The TANC2 gene, encodes a synaptic scaffolding protein that interacts with other proteins at the postsynaptic density to regulate dendritic spines and excitatory synapse formation. The TANC2 gene mutations were reported in neurodevelopmental disorders and epilepsy but not in LGS ever.

Case presentation: Here we describe the case of a boy with LGS who presented with multiple seizure patterns, such as myoclonic, atonic, atypical absence, generalized tonic-clonic, focal seizures, and notable cognitive and motor regression. The seizures were refractory to many antiepileptic drugs. He got seizure-free with ketogenic diet combined with antiepileptic drugs. A de novo nonsense mutation c.4321C>T(p.GIn1441Ter) in TANC2 gene was identified by the whole-exome sequencing and confirmed by Sanger sequencing.

Conclusion: We described the first Chinese case with LGS associated to a de novo nonsense mutation c.4321C > T(p. GIn1441Ter) in TANC2 gene, which would expand the clinical spectrum related to TANC2 mutations and contribute to better understanding of genotype-phenotype relationship to guide precision medicine.
\end{abstract}

Keywords: TANC2, Epilepsy, Lennox-Gastaut syndrome, Case report

\section{Background}

Lennox-Gastaut syndrome (LGS) is a severe epileptic encephalopathy in which the epileptiform abnormalities may contribute to progressive dysfunctions [1], characterized by polymorphic seizures and neuropsychological decline [2]. LGS may arise from multiple etiologies. In symptomatic cases, LGS most frequently occurs secondary to damage to the brain resulting from prenatal or perinatal insult, infection, malformations, or tumor [3]. However, approximately one fourth to one third of

\footnotetext{
*Correspondence: gzchcwx@126.com; chenwenxiong@gwcmc.org

†Yang Tian, Zhen Shi and Chi Hou contributed equally to this work. Department of Neurology, Guangzhou Women and Children's Medical Center, Guangzhou Medical University, 9\# Jin Sui Road, 510623 Guangzhou, Guangdong Province, PR China
}

LGS cases have no clear etiology, among which some cases were found to be associated with genetic mutations thanks to increased application of next-generation sequencing in the field of clinical diagnosis.

A previous study systematically reported the genetic and clinical data for 20 patients with pathogenic TANC2 mutations for the first time in 2019, as a follow-up of a recent meta-analysis of whole-exome sequencing (WES) data from 10,927 families with neurodevelopmental disorders (NDDs), which include autism spectrum disorder (ASD) and epilepsy [4]. The TANC2 gene, located at chromosome $17 \mathrm{q} 23.2$-q23.3, encodes a synaptic scaffolding protein that interacts with other proteins at the postsynaptic density to regulate dendritic spines and excitatory synapse formation [5]. However, it is unknown 

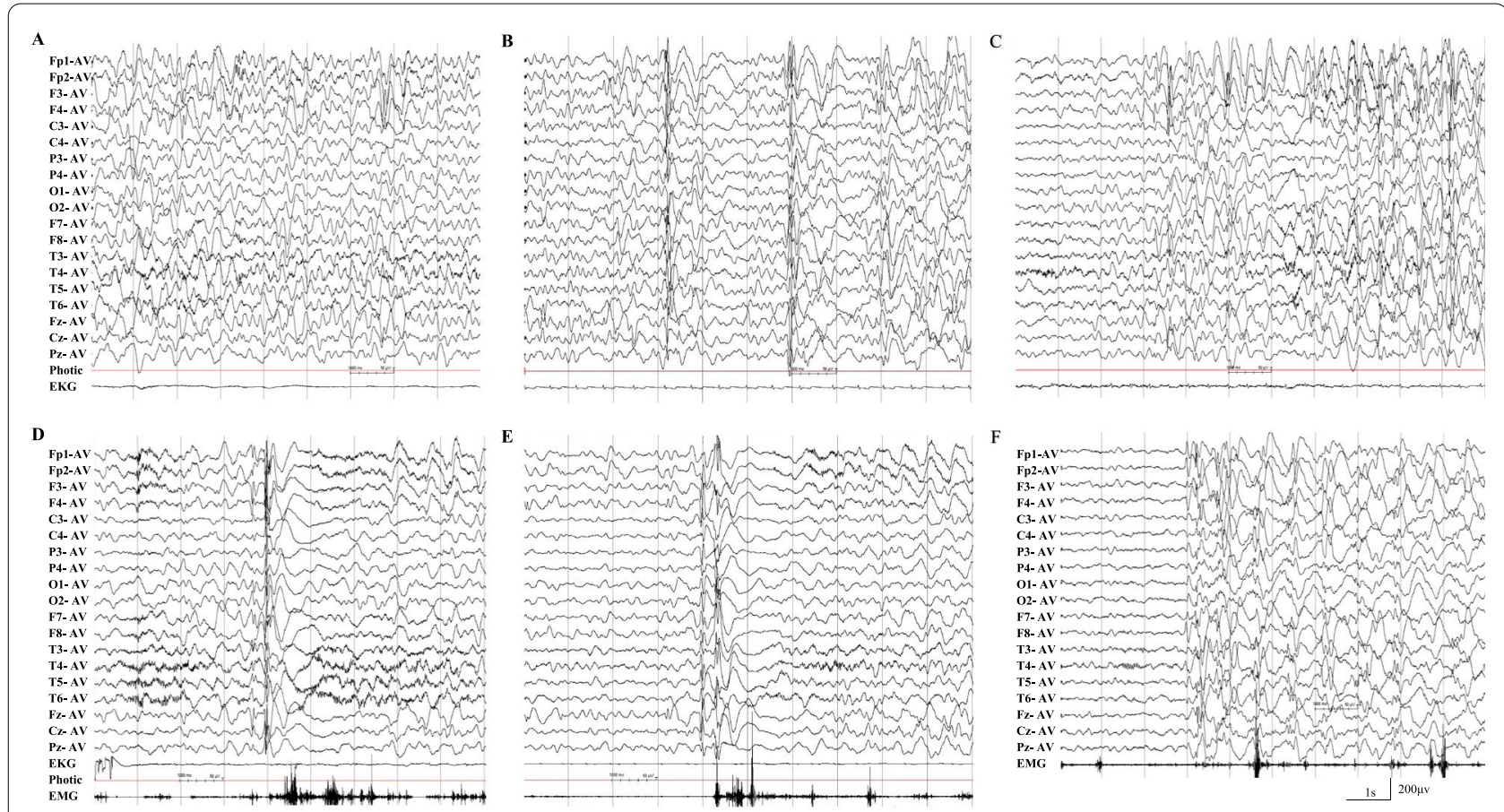

Fig. 1 The EEG of the patient with TANC2 mutation. A The background was slow, diffused $\theta$ and $\delta$ activities were seen; B Generalized slow spike-and-wave complexes during sleep; $\mathbf{C}$ Paroxysmal generalized 1.5-2 $\mathrm{Hz}$ spike-and-wave complexes with atypital absence seizure; $\mathbf{D}$ Generalized spike-and-wave complexes with atonic seizure and flattening in EMG; $\mathbf{E}$ Generalized spike-and-wave complexes with clonic seizure and EMG burst; FThe re-checked EEG, generalized slow spike-and-wave complexes during sleep

whether TANC2 mutations are associated with epileptic encephalopathy.

The present study describes a 1-year and 11-month old boy who suffered from LGS. By WES analysis, a de novo nonsense mutation was identified in TANC2 and thus established the link between LGS and TANC2 mutations.

\section{Case presentation}

The proband was a 1-year and 11-month old boy at the time of admission. He is the third child with two healthy elder brothers of a non-consanguineous marriage, born after full-term by normal delivery, with a birth weight of $3000 \mathrm{~g}$ and allergy history of cephalosporin. No other members in the family have seizures. Nothing abnormal was observed in the perinatal period. Motor development and language development were mildly delayed before the onset of seizures, with an unbalanced gait and dual tone. The first two seizure episodes occurred within half a day when the temperature reached $39^{\circ} \mathrm{C}$ at 1-year and 10-month old, which presented with generalized tonic-clonic, lasting for about $2 \mathrm{~min}$ per episode. Subsequently, the convulsion occurred twice during the following week without fever or other inducements. Twenty days later, the convulsion occurred again and presented as tonic of four limbs. Then the convulsion occurred more frequently (10-20/day) with varied epileptic seizure types such as myoclonic, atypical absence, atonic seizures a week before admission into our clinical center.

His height, body weight, and head circumference were $85.0 \mathrm{~cm}, 11.8 \mathrm{~kg}$, and $48.0 \mathrm{~cm}$ respectively at the admission. He had no obvious craniofacial dysmorphisms with fontanel closure. No other positive signs were found upon physical examination except slight gait instability. Results from routine examination including urine/ blood/cerebrospinal fluid, blood/cerebrospinal fluid biochemistry, blood electrolytes examination, blood glucose, cerebrospinal fluid pathogens, metabolic screening and brain magnetic resonance imagery were normal. VideoEEG monitoring was performed for $3 \mathrm{~h}$ which showed slow background activity accompanied by a $2 \sim 3 \mathrm{~Hz}$ slow activity major in anterior area (Fig. 1A), interictal EEG showed a large number of generalized high amplitude $2 \sim 2.5 \mathrm{~Hz}$ slow spike-and-wave complexes (Fig. 1B). Two atypical absence seizures with generalized high amplitude $2.5 \sim 3 \mathrm{~Hz}$ spike-and-wave complexes lasting $7 \mathrm{~s}$ (Fig. 1C); two atonic seizures with $1-2 \mathrm{~Hz}$ polyspikesand-wave complexes and electromyography flat (Fig. 1D); and several myoclonic seizures with $1-2 \mathrm{~Hz}$ polyspikesand-wave complexes and electromyography burst were recorded (Fig. 1E). The myoclonic seizures disappeared 


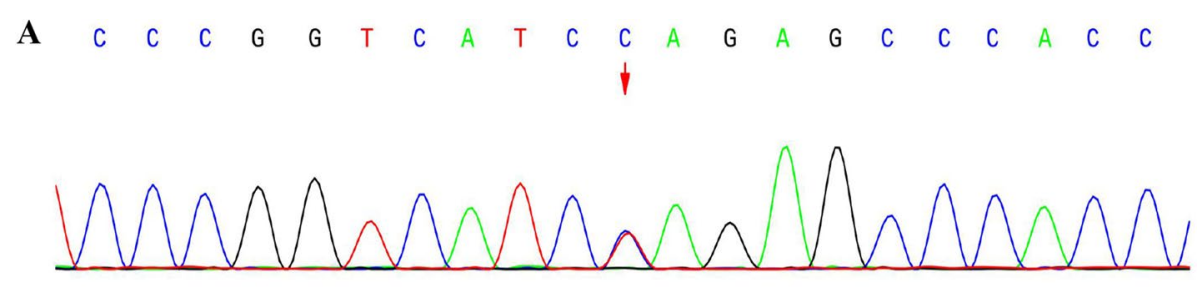

B

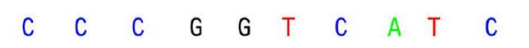

c

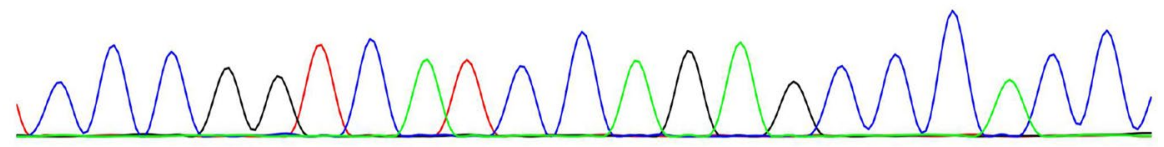

C

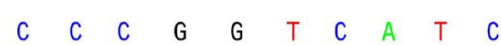
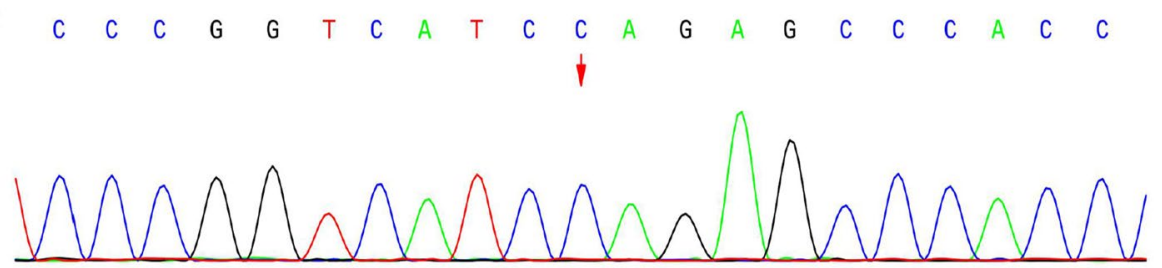

Fig. 2 Sequence analysis of the TANC2 gene in the family. The arrows show the mutation site, Heterozygous mutation of TANC2 gene C.4321C > T ( $p . G \ln 1441$ Ter) was identified in the proband, none the parents carried the mutation. A The result of the proband; $\mathbf{B}$ The result of the father; $\mathbf{C}$ The result of the mother

and atypical absence and atonic seizures improved with combined treatment of sodium valproate and nitrazepam. Then focal and general tonic-clonic seizures were seen during sleep occasionally. Follow-up EEG showed a large number of generalized high amplitude $1.5 \sim 2.5 \mathrm{~Hz}$ slow spike-and-wave complexes in the slow background (Fig. 1F). Lamotrigine then was added as the third drug for combination therapy, and after which the focal and general tonic-clonic seizures were well controlled and atypical absence, atonic seizures reduced. However, subsequently epileptic spasms emerged more than 10 times a day especially when he was quiet or shortly after waking up. During the two-month follow-up, the child exhibited gait instability and cognition regression, the Gesell scale scored 55 , including gross motor 54 , fine motor 47 , language 70, cognition 50, social development 54. Based on the aforementioned clinical manifestations, the patient was diagnosed with Lennox-Gastaut syndrome (LGS). He was seizure-free with ketogenic diet combined with antiepileptic drugs forementioned finally. The blood Ketogen level was maintained at about $3 \mathrm{mmol} / \mathrm{L}$.

To identify the potential pathogenic mutations, the proband and the parents' genomic DNA was extracted from peripheral blood lymphocytes using the QIAamp ${ }^{\circledR}$ Blood Mini Kit (Qiagen, Hilden, Germany) and subjected to whole-exome sequencing (WES) on Illumina NovaSeq6000 (Illumina, San Diego, CA, USA). The read mapping, variant calling, genome annotation, and variant prioritization were performed as described previously [6], the variant was classified according to standards and guidelines of the American College of Medical Genetics and Genomics (ACMG) [7]. The results were also confirmed by Sanger sequencing. WES showed only one de novo heterozygous mutation c.4321C $>\mathrm{T}$ (p.Gln1441Ter) in TANC2 gene (NM_025185.4) in the proband (Fig. 2). No other rare variants in any other genes associated with LGS reported previously were found in the patient. This nonsense mutation would lead to premature translation termination and has no allele frequency in several commonly used databases (i.e., dbSNP, gnomAD, ClinVar, HGMD). The in silico prediction score of GERP (Genomic Evolutionary Rate Profiling), LRT (Likelihood ratio test) and Mutation Taster is $5.29,0$ and 1 respectively which indicate pathogenicity. According to the ACMG clinical variant interpretation guidelines, the pathogenic evaluation of this variant was "PVS1 + PS2 + PM2", which classified as "Pathogenic". The genetic characteristics and pathogenetic analysis of the variant in TANC2 were displayed in Table 1. 
Table 1 The genetic characteristics and pathogenetic analysis of TANC2 mutation identified in this study

\begin{tabular}{lllllllll}
\hline Gene & Location & Nucleotide alteration & Mutation type & MAF & GERP & LRT & MT & ACMG values \\
\hline TANC2 & chr17:63420303 & C.4321C>T & Nonsense & NA & 5.29 & 0 & 1 & PVS1+PS2+PM2
\end{tabular}

MAF Minor allele frequency, GERP Genomic Evolutionary Rate Profiling, LRT Likelihood ratio test, MT Mutation Taster, NA Not available, ACMG American College of Medical Genetics and Genomics

\section{Discussion and conclusions}

Lennox-Gastaut Syndrome (LGS) is considered as a severe epileptic encephalopathy with onset in childhood, which implies that the epileptic activity contributes to mental problems and behavioral disorders [8]. It is defined by a triad of multiple drug-resistant seizure types, a specific interictal EEG pattern showing bursts of slow spike-wave complexes or generalized paroxysmal fast activity, and intellectual disability [8]. However, not all patients have all of the core seizure types (i.e., tonic, atonic, and atypical absences), especially at the onset [9]. The age of onset of LGS is typically between 1 and 7 years, most commonly between 3 and 5 years [10]. The long-term outcome for patients with LGS is generally poor and complete seizure freedom is unusual [8]. Its etiology is broken into six subgroups [11], among which genetic factors are getting more and more attention, where more than ten related pathogenic genes have been reported.

In the patient reported here, febrile seizures occurred twice at 1-year and 10-month old. Since then, he manifested frequent seizures and exhibited multiple seizure patterns including atypical absence, atonic, myoclonic, focal, general tonic-clonic and tonic. The video EEG revealed synchronous (generalized) discharge, with a large number of generalized high amplitude $2 \sim 2.5 \mathrm{~Hz}$ slow spike-and-wave complexes, spike-and-wave complexes, polyspikes-and-wave complexes in the background. The oral administration of sodium valproate, nitrazepam and lamotrigine made myclonic, focal and general tonic-clonic seizures free and reduced the frequency of atypical absence and atonic seizures, but epileptic spasms emerged frequently later, which indicated that it was a refractory epilepsy and had a partial response to sodium valproate combined with nitrazepam and lamotrigine treatment. The child developed mild delay in motor and speech before the onset of seizures. His cognitive and motor function regressed with the refractory epilepsy in 2 months follow-up. All these symptoms were consistent with LGS diagnosis.

There were no abnormalities in brain magnetic resonance imaging. Metabolic screening and cerebrospinal fluid etiology screening were both negative. A de novo c.4321C $>\mathrm{T}$ (p.Gln1441Ter) mutation in TANC2 gene was identified in the child by whole-exome sequencing and confirmed by Sanger sequencing. The variant is located in a high conserved region and has a deleterious effect by in silico prediction. According to the ACMG clinical variant interpretation guidelines, the variant is classified as a pathogenic mutation. We considered the variant as the cause of the disease in this patient based on the genotype-phenotype association and its co-segregation with the disease.

\section{TANC2 function, clinical manifestations, and precision medicine}

Our study and the review of the literature suggested an important role for TANC2 in brain development. TANC2 encodes a synaptic scaffolding protein that interacts with other proteins, especially PSD-95 (postsynaptic density-95), KIF1A (the primary motor protein for synaptic vesicles), and various subunits of the NMDA receptor (Grin1 and Grin2B), at the postsynaptic density to regulate dendritic spines and excitatory synapse formation $[12,13]$. In addition, TANC2 deficiency leads to embryonic lethality, indicating the importance of TANC2 during embryonic development [12]. According to the transcriptomic profiles in the developing human cerebral cortex, TANC2 is broadly expressed in many cell types but enriched in excitatory neurons and radial glia [4]. These functional investigations are consistent with our observation that truncating mutations in our report and others lead to malfunction in synapse-related neurological processes.

We observed severe epilepsy in our case, however, within a larger pool of 20 probands or affected siblings with TANC2 disruptive variants, epilepsy was only observed for 11 individuals and the degree of epilepsy was variable [4]. Upon more careful evaluation of the individual mutations and their corresponding clinical manifestations, there was no apparent genotype-phenotype association, even though all the likely disruptive mutations lead to the missing of PDZ interacting motif, which is essential for its synaptic localization [4, 14]. The most consistent phenotypes associated with TANC2 disruption, including intellectual disability (ID), speech-language delay, and childhood motor delay, are also observed in our case. Considering the still limited number of mutations reported so far, more reports in the future would be needed to expand the clinical spectrum related to TANC2 mutations and to clarify the genotypephenotype correlations (if any). 
We also reported the treatment and the response of our patient in this case. Again only with increased number of future reports and thus accumulated database on genotype-phenotype relationships and corresponding drug response, can we start to realize the ultimate goal of precision medicine.

In conclusions, we performed a clinical and molecular genetic study related to a Chinese boy with LennoxGastaut Syndrome and found a potential causative mutation in TANC2. After reviewing the literature about the TANC2 gene and function, we demonstrated that the gene plays an important role in neurodevelopmental disorder and epilepsy. To the best of our knowledge, this is the first report about TANC2 mutation in LGS patients, which would increase clinicians' understanding of the genetic profile of LGS and expand the still limited TANC2 mutation spectrum as well.

\begin{abstract}
Abbreviations
ACMG: American College of Medical Genetics and Genomics; ASD: Autism spectrum disorder; EEG: Electroencephalogram; HGMD: The Human Gene Mutation Database; ID: Intellectual disability; LGS: Lennox-Gastaut syndrome; LRT: Likelihood ratio test; NDDs: Neurodevelopmental disorders; NMDA: N-methyl-D-aspartate; PSD: Postsynaptic density; WES: Whole-exome sequencing.
\end{abstract}

\section{Acknowledgements}

The authors are very grateful to the participating family for their courtesy and humanity, as well as very helpful advice from Dr. Mingwei Huang from Aegicare (Shenzhen) Technology Co., Ltd.

\section{Authors' contributions \\ Study Design, Analysis and Revision of the Manuscript, WC; Follow-up of Patient's Information, YT, CH and WL; Original Draft Preparation, YT, ZS and $\mathrm{CH}$; Reanalysis of WGS Data, ZS; Collection of Clinical and WGS Data, YT, XW, $H Z$, and $X L$; Literature Search and Sanger Sequencing, XW, WL, HZ and YT. All authors have read and agreed to the published version of the manuscript.}

\section{Funding}

This work was funded by the Internal Fund of the pediatric Institute of Guangzhou Women and Children's Medical Center (grant number:YIP-2019028 and 1600050-04), the Science and Technology Program of Guangzhou (grant number: 202102020748), China. These funders had role in the design of the study and collection, analysis, and interpretation of data and in writing the manuscript.

\section{Availability of data and materials}

All data and materials mentioned in this manuscript, except patient's private information, can be promptly available to readers by a reasonable request to the corresponding author.

\section{Declarations}

Ethics approval and consent to participate

This research was approved by the Ethics Committee of Guangzhou Women and Children's Medical Center, conforming to the Helsinki Declaration. Informed written consent for clinical and genetic analyses was obtained from the patient's parents.

\section{Consent for publication}

Written informed consent was obtained from the parents for publication of this case report and any accompanying tables/images.

\section{Competing interests}

The authors declare that they have no competing interests.

Received: 8 May 2021 Accepted: 23 November 2021

Published online: 03 December 2021

\section{References}

1. Engel J Jr. International league against E. a proposed diagnostic scheme for people with epileptic seizures and with epilepsy: report of the ILAE task force on classification and terminology. Epilepsia. 2001;42(6):796-803.

2. Bourgeois BF, Douglass LM, Sankar R. Lennox-Gastaut syndrome: a consensus approach to differential diagnosis. Epilepsia. 2014;55(Suppl 4):4-9.

3. van Rijckevorsel K. Treatment of Lennox-Gastaut syndrome: overview and recent findings. Neuropsychiatr Dis Treat. 2008;4(6):1001-19.

4. Guo H, Bettella E, Marcogliese PC, Zhao R, Andrews JC, Nowakowski TJ, et al. Disruptive mutations in TANC2 define a neurodevelopmental syndrome associated with psychiatric disorders. Nat Commun. 2019;10(1):4679.

5. Tassano E, Accogli A, Ronchetto P, Tortora D, Tavella E, Gimelli G, et al. $17 q 23.3$ de novo microdeletion involving only TANC2 gene: a new case. Eur J Med Genet. 2020;63(12):104094.

6. Schubert J, Siekierska A, Langlois M, May P, Huneau C, Becker F, et al. Mutations in STX1B, encoding a presynaptic protein, cause fever-associated epilepsy syndromes. Nat Genet. 2014;46(12):1327-32.

7. Richards S, Aziz N, Bale S, Bick D, Das S, Gastier-Foster J, et al. Standards and guidelines for the interpretation of sequence variants: a joint consensus recommendation of the American College of Medical Genetics and Genomics and the Association for Molecular Pathology. Genet Med. 2015;17(5):405-24

8. Camfield PR. Definition and natural history of Lennox-Gastaut syndrome. Epilepsia. 2011;52(Suppl 5):3-9.

9. Arzimanoglou A, French J, Blume WT, Cross JH, Ernst JP, Feucht M, et al. Lennox-Gastaut syndrome: a consensus approach on diagnosis, assessment, management, and trial methodology. Lancet Neurol. 2009;8(1):82-93.

10. Arzimanoglou A, Resnick T. All children who experience epileptic falls do not necessarily have Lennox-Gastaut syndrome... But many do. Epileptic Disord. 2011;13(Suppl 1):S3-13.

11. Scheffer IE, Berkovic S, Capovilla G, Connolly MB, French J, Guilhoto $L$, et al. ILAE classification of the epilepsies: position paper of the ILAE Commission for Classification and Terminology. Epilepsia. 2017:58(4):512-21.

12. Han S, Nam J, Li Y, Kim S, Cho SH, Cho YS, et al. Regulation of dendritic spines, spatial memory, and embryonic development by the TANC family of PSD-95-interacting proteins. J Neurosci. 2010;30(45):15102-12.

13. Stucchi R, Plucinska G, Hummel JJA, Zahavi EE, Guerra San Juan I, Klykov O, et al. Regulation of KIF1A-driven dense Core vesicle transport: $\mathrm{Ca}\left({ }^{2+}\right) / \mathrm{CaM}$ controls DCV binding and Liprin-alpha/TANC2 recruits DCVs to postsynaptic sites. Cell Rep. 2018;24(3):685-700.

14. Gasparini A, Tosatto SCE, Murgia A, Leonardi E. Dynamic scaffolds for neuronal signaling: in silico analysis of the TANC protein family. Sci Rep. 2017;7(1):6829.

\section{Publisher's Note}

Springer Nature remains neutral with regard to jurisdictional claims in published maps and institutional affiliations. 